\title{
Ensemble Learning Model for Petroleum Reservoir Characterization: A Case of Feed-Forward Back-Propagation Neural Networks
}

\author{
Fatai Anifowose ${ }^{1}$, Jane Labadin ${ }^{1}$, and Abdulazeez Abdulraheem ${ }^{2}$ \\ ${ }^{1}$ Faculty of Computer Science and Information Technology, Universiti Malaysia Sarawak \\ fanifowose@gmail.com, ljane@fit.unimas.edu.my \\ ${ }^{2}$ Department Petroleum Engineering, King Fahd University of Petroleum and Minerals \\ aazeez@kfupm.edu.sa
}

\begin{abstract}
Conventional machine learning methods are incapable of handling several hypotheses. This is the main strength of the ensemble learning paradigm. The petroleum industry is in great need of this new learning methodology due to the persistent quest for better prediction accuracies of reservoir properties for improved exploration and production activities. This paper proposes an ensemble model of Artificial Neural Networks (ANN) that incorporates various expert opinions on the optimal number of hidden neurons in the prediction of petroleum reservoir properties. The performance of the ensemble model was evaluated using standard decision rules and compared with those of ANN-Ensemble with the conventional Bootstrap Aggregation method and Random Forest. The results showed that the proposed method outperformed the others with the highest correlation coefficient and the least errors. The study also confirmed that ensemble models perform better than the average performance of individual base learners. This study demonstrated the great potential for the application of ensemble learning paradigm in petroleum reservoir characterization.
\end{abstract}

Keywords: Ensemble, artificial neural networks, hidden neurons, reservoir characterization, porosity, permeability.

\section{Introduction}

The degree of application of Computational Intelligence (CI) in petroleum engineering over the years is an indication of the relative increase in the level of awareness and interest in the concept [1]. Gradually, researchers in the petroleum industry have moved from the use of empirical correlations and linear regression models to the use of CI [2]. Interestingly, the application of CI in the industry has majorly been limited to Artificial Neural Networks (ANN) [3]. However, ANN has a number of deficiencies [4]. Recently, the interest of CI researchers in hybrids and ensembles of these techniques has increased. Reports have showed that the performance of hybrid CI models is better than that of each of its components [5 - 7] while ensemble models have not been applied in oil and gas reservoir characterization. 